\title{
The Laplacian on a random one-dimensional lattice
}

\author{
E J Gardner $\uparrow$, C Itzykson and B Derrida \\ Cen-Saclay, 91191 Gif-sur-Yvette Cedex, France
}

Received 3 June 1983, in final form 10 October 1983

\begin{abstract}
We study the spectrum of (minus) the Laplacian on a random one-dimensional lattice. It extends monotonically throughout the positive real axis with a continuum limit behaviour for small values. We find as expected localisation effects throughout the whole range. We use a variety of techniques which are shown to be consistent and in agreement with a Monte Carlo simulation. They include small and large disorder expansions, direct integral equations for probability distributions as well as the replica method. The latter is also used to investigate Green functions.
\end{abstract}

\section{Introduction}

To test the recent proposal by Christ et al (1982a, b) of using a random lattice instead of a regular one, as a substitute for continuous space-time in strong coupling field theory, we investigate here the one-dimensional case. We concentrate on free fields or equivalently on Brownian motion. Although this example is rather simple, it is also quite instructive. It illustrates the difficulties and possible limitations of the use of such random lattices. The price paid for translational (and rotational) symmetry restoration is of course the necessity of performing averages. Under 'experimental' situations this might turn out to be a quite formidable task. A measurement on a large specimen would presumably require an appreciation of the full probability distribution of whatever random variable (spectrum, Green function . . .) is considered. To put it differently, some quantities naturally equal on a regular lattice might turn out to be different in the random case due to different averaging procedures.

New phenomena appear due to the disordered structure of the lattice. The most obvious one is localisation, i.e. a decay of 'free' wavefunctions at large distances. This is mostly apparent in the one-dimensional case, where this decay is exponential and measured in terms of a localisation length. Another aspect is that disorder induces interactions. If we think in terms of Brownian motion, we expect some short-distance effective attractive interaction between two particles on a random lattice. In other terms the average of products of propagators is not the product of averages. It is clear that a complete understanding of these disturbing effects is required in order to disentangle them from the study of genuine interactions if one would like to apply the method to problems like lattice gauge theories, for instance.

Fortunately in one dimension it is possible to go rather far in this analysis. Numerous previous works have been devoted to similar problems. We may quote for instance the study of a chain of random springs by Dyson (1953), and the investigation of a one-dimensional Schrödinger equation in a random potential by Halperin (1965). A review is given by Alexander et al (1981).

† Supported by a Fellowship from the Royal Society. 
Our plan is the following. In $\S 2$ we state the problem and establish the basic equations enabling one to study the spectrum of the random Laplacian. In $\S \S 3$ and 4 we investigate approximations valid for small and large eigenvalues. Slightly improperly we call these eigenvalues frequencies and denote them by $\Omega$. In $\$ 5$ we compare these expansions with data obtained from a numerical simulation. Good agreement is found for small and large $\Omega$. The random spectrum is a smooth function behaving as $\Omega^{-1 / 2}$ for small values and as $\Omega^{-2}$ for large values.

In $\S 6$ we present some analysis of the original equations, showing that they can be solved recursively in terms of integrals. In $\S 7$ we study an alternative method, the so-called replica trick, related to the original equations by an appropriate Fourier transformation. The results are in agreement with those of Stephen and Kariotis (1982) who also used the replica method and with the results of previous sections. We then extend the work of Stephen and Kariotis to calculate Green functions at non-zero separations.

\section{Derivation of the equations}

In a previous paper (Itzykson 1983) we have discussed the free field equations in arbitrary dimensions following the prescriptions of Christ et al (1982). Let us just recall that the sites are randomly distributed in space and the lattice is constructed according to the prescriptions of Christ et al (1982). In one dimension we are left with the Laplacian on scalar fields

$$
\frac{1}{\sigma_{i}} \sum_{\substack{\text { nearest } \\ \text { neighbours } j}} \sigma_{i j} \frac{\left(\varphi_{j}-\varphi_{i}\right)}{l_{i j}} .
$$

In one dimension $\sigma_{i j}$ is equal to one, $l_{l}$ is the distance between neighbours and $\sigma_{t}$ the volume of a cell, i.e. $\left(l_{i, 1+1}+l_{i-1, t}\right) / 2$ in one dimension. We will simplify the problem by setting $\sigma_{i}$ equal to its mean value which we take as unit of length. This will not change any essential feature of the results. The points may then be ordered along the line. Their separations $l_{l, t+1} \equiv l_{i+1}$ are independent Poissonian variables with mean one, i.e.

$$
p\left(l_{\alpha}, l_{\beta}, \ldots\right) \mathrm{d} l_{\alpha} \mathrm{d} l_{\beta} \ldots=\exp \left[-\left(l_{\alpha}+l_{\beta}+\ldots\right)\right] \mathrm{d} l_{\alpha} \mathrm{d} l_{\beta} \ldots
$$

The eigenvalue equation for the Laplacian is written

$$
\left(\varphi_{n+1}-\varphi_{n}\right) / l_{n}-\left(\varphi_{n}-\varphi_{n-1}\right) / l_{n-1}=-\Omega \varphi_{n} .
$$

The spectrum corresponds to $\Omega>0, \Omega$ having the dimension (length) ${ }^{-2}$ when restoring the units.

The average density of states per unit length, and unit frequency range, is denoted $\rho(\Omega)$. The existence of an ultraviolet cut-off is reflected in the normalisation

$$
\int_{0}^{\infty} \mathrm{d} \Omega \rho(\Omega)=1 .
$$

Rather than solving (3) directly, we consider a similar equation with $-\Omega$ replaced by $\omega>0$. Introducing new variables $Q_{n}=\left(\varphi_{n+1}-\varphi_{n}\right) / l_{n}$ gives the equation

$$
Q_{n+1}-2 Q_{n}+Q_{n-1}=\omega l_{n} Q_{n} .
$$

Therefore $Q_{n}$ satisfies Laplace's equation on a regular lattice, labelled by the index 
of the sites $n$, with a potential term $\omega l_{n} Q_{n}$. At sufficiently large $n$ the $Q_{n}$ 's will grow exponentially with $n$

$$
Q_{n} \sim \mathrm{e}^{\gamma(\omega) n}
$$

with a positive Liapunov exponent $\gamma(\omega)$. If initial conditions are specified at $n=0,1$, then $Q_{n}$ is a polynomial of degree $n$ in $\omega$ with roots at values $-\Omega_{s}$ which in the limit $n \rightarrow \infty$ correspond to the eigenvalues in (3). Therefore

$$
Q_{n} \underset{n \rightarrow \infty}{\sim} \prod_{s}\left(\omega+\Omega_{s}\right)=\exp \sum_{s=1}^{\mathrm{n}} \ln \left(\omega+\Omega_{s}\right)
$$

and so from Dyson (1953), Thouless (1972)

$$
\gamma(\omega)=\int_{0}^{\infty} \mathrm{d} \Omega \rho(\Omega) \ln (\omega+\Omega), \quad \rho(\Omega)=\frac{1}{\pi} \operatorname{Im}_{\varepsilon \rightarrow 0} \frac{\mathrm{d} \gamma}{\mathrm{d} \omega}(-\Omega-\mathrm{i} \varepsilon) .
$$

The second-order linear equation (5) may be transformed to a first-order nonlinear one by making a change of variable $R_{n}=Q_{n+1} / Q_{n}$, with $R_{n}$ satisfying

$$
R_{n}+R_{n-1}^{-1}=2+\omega l_{n}
$$

At large $n$, from $(6), R_{n}$ behaves as $\mathrm{e}^{\gamma(\omega)}$ and so

$$
\gamma(\omega)=\lim _{n \rightarrow \infty}\left\langle\ln R_{n}\right\rangle, \quad \rho(\Omega)=\left.\frac{1}{\pi} \operatorname{Im} \frac{\mathrm{d}}{\mathrm{d} \omega} \lim _{n \rightarrow \infty}\left\langle\ln R_{n}\right\rangle\right|_{\omega=-\Omega-\mathrm{i} \varepsilon} .
$$

Here the brackets mean an average over the probability distribution of the separations $l$.

It is possible to derive an integral equation for $P_{n}\left(R_{n}\right)$, where $P_{n}\left(R_{n}\right) \mathrm{d} R_{n}$ is the probability that the random variable $R_{n}$ has value between $R_{n}$ and $R_{n}+\mathrm{d} R_{n}$. We set $R_{0}=1$. The $l$ 's are independent and $R_{n-1}$ depends only on $l_{i}, i<n$. Consequently $l_{n}$ and $R_{n-1}$ are also independent. From (9)

$$
P_{n}\left(R_{n}\right)=\int_{1}^{\infty} \mathrm{d} R_{n-1} P_{n-1}\left(R_{n-1}\right) \int_{0}^{\infty} \mathrm{d} l \mathrm{e}^{-l} \delta\left(R_{n}+R_{n-1}^{-1}-2-\omega l\right) .
$$

As $n$ goes to infinity, $P_{n}$ tends to a fixed distribution $P$. One can check that for $\omega>0$, this distibution is concentrated on $[1, \infty]$, see equation (9). Thus

$$
\begin{aligned}
P(R)=\int_{1}^{\infty} & \mathrm{d} R^{\prime} P\left(R^{\prime}\right) \int_{0}^{\infty} \mathrm{d} l \mathrm{e}^{-l} \delta\left(R+R^{\prime-1}-2-\omega l\right) \\
& =\int_{1}^{\infty} \frac{\mathrm{d} R^{\prime}}{\omega} P\left(R^{\prime}\right) \exp \left(\frac{-1}{\omega\left(R+R^{\prime-1}-2\right)}\right) \theta\left(R+R^{\prime-1}-2\right) .
\end{aligned}
$$

Equations (11) and (12) easily generalise to an arbitrary distribution $p(l) \mathrm{d} l$. What makes equation (12) non-trivial is the presence of a $\theta$ function. Otherwise it would readily be solved. The recurrence relation (11) implies that (12) must admit a positive normalisable density of probability as a solution. We postpone to $\S 6$ a detailed analysis of this equation. From $(10)$ the index $\gamma(\omega)$ is

$$
\gamma(\omega)=\int_{1}^{\infty} \mathrm{d} R P(R) \ln R .
$$

Before considering the random case we first determine the density $\rho$ for a regular lattice with unit spacing. In this case $P(R)=\delta(R-A)$ where $A$ is the limit of $R_{n}$, 
solution of (9) with $l_{n}=1$, i.e. the largest root of

Therefore

$$
A^{2}-2(1+\omega / 2) A+1=0, \quad A=1+\omega / 2+\left(\omega+\omega^{2} / 4\right)^{1 / 2} .
$$

$$
\begin{aligned}
& \gamma_{0}(\omega)=\ln \left[1+\omega / 2+\left(\omega+\omega^{2} / 4\right)^{1 / 2}\right], \\
& \rho_{0}(\Omega)=\frac{1}{2 \pi[\Omega(1-\Omega / 4)]^{1 / 2}}=\frac{\theta(\Omega) \theta(4-\Omega)}{2 \pi} \frac{1}{\sqrt{\Omega}}\left(1+\frac{\Omega}{8}+O\left(\Omega^{2}\right)\right) .
\end{aligned}
$$

On the regular lattice the spectrum is of course restricted to a finite band $0<\Omega<4$, corresponding to a Brillouin zone in momentum space, and for small $\Omega, \rho_{0}$ has the continuum limit behaviour $(2 \pi)^{-1} / \sqrt{\Omega}$. The integral of the latter is of course divergent corresponding to an infinite number of modes per unit.volume.

\section{Weak disorder-low frequency expansion}

In order to solve (9) in the random case, we artificially introduce a parameter $\lambda$, measuring the deviation of the lattice from a regular one. It turns out that the corresponding expansion is useful for small $\omega$ (Derrida and Orbach 1983), the large wavelength limit being weakly sensitive to the local disorder. We set

$$
l_{n}=1+\lambda z_{n}, \quad R_{n}=A_{n} \exp \left(\lambda B_{n}+\lambda^{2} C_{n}+\lambda^{3} D_{n}+\lambda^{4} E_{n}+\ldots\right),
$$

where $\lambda$ will later be set equal to one. Since $\left\langle l_{n}\right\rangle=1$ we have $\left\langle z_{n}\right\rangle=0$ and the regular lattice result $R_{n} \rightarrow A$ as $n \rightarrow \infty$ is obtained for $\lambda=0$. Of course $A$ is given by (14). Let $\langle B\rangle,\langle C\rangle,\langle D\rangle,\langle E\rangle \ldots$ be the averages of $B_{n}, C_{n}, D_{n}, E_{n}, \ldots$ Then

$$
\gamma(\omega)=\gamma_{0}(\omega)+\lambda\langle B\rangle+\lambda^{2}\langle C\rangle+\lambda^{3}\langle D\rangle+\lambda^{4}\langle E\rangle+\ldots
$$

Substituting the expansion (16) into (9), we have for large $n, A_{n} \rightarrow A$, and using (14)

$$
\begin{aligned}
A^{2}\left[\operatorname { e x p } \left(\lambda B_{n}\right.\right. & \left.\left.+\lambda^{2} C_{n}+\ldots\right)-1\right] \\
& \left.=\lambda(A-1)^{2} z_{n}-\left[\exp -\left(\lambda B_{n-1}+\lambda^{2} C_{n-1}+\ldots\right)-1\right)\right] .
\end{aligned}
$$

This leads to the following recursion relations for the coefficients:

(a)

$A^{2} B_{n}=(A-1)^{2} z_{n}+B_{n-1}$,

$$
\begin{aligned}
& A^{2}\left(C_{n}+\frac{1}{2} B_{n}^{2}\right)=C_{n-1}-\frac{1}{2} B_{n-1}^{2}, \\
& A^{2}\left(D_{n}+B_{n} C_{n}+\frac{1}{6} B_{n}^{3}\right)=D_{n-1}-B_{n-1} C_{n-1}+\frac{1}{6} B_{n-1}^{3}, \\
& A^{2}\left(E_{n}+B_{n} D_{n}+\frac{1}{2} C_{n}^{2}+\frac{1}{2} B_{n}^{2} C_{n}^{-}+\frac{1}{24} B_{n}^{4}\right) \\
& \quad=E_{n-1}-B_{n-1} D_{n-1}-\frac{1}{2} C_{n-1}^{2}+\frac{1}{2} B_{n-1}^{2} C^{n-1}-\frac{1}{24} B_{n-1}^{4} .
\end{aligned}
$$

Equations (18) and (19) show the relation between a small $(A-1)$, i.e. $\omega$, and the $\lambda$ expansion. By taking averages of equations (19) and of their products, using the fact that $B_{n-1}, C_{n-1} \ldots$, depend on $z_{p}, p<n$, but are independent of $z_{n}$, and that the averages do not depend on $n$, we obtain expressions for $\langle B\rangle,\langle C\rangle, \ldots$ :

$$
\begin{aligned}
& \langle B\rangle=0, \quad\langle C\rangle=-\frac{1}{2}\left\langle\left(\frac{A-1}{A+1} z\right)^{2}\right\rangle, \quad\langle D\rangle=\frac{1}{3}\left\langle\left(\frac{A-1}{A+1} z\right)^{3}\right\rangle \\
& \langle E\rangle=-\frac{1}{4}\left\langle\left(\frac{A-1}{A+1} z\right)^{4}\right\rangle-\frac{1}{2} \frac{3+2 A^{2}}{A^{4}-1}\left[\left\langle\left(\frac{A-1}{A+1} z\right)^{2}\right\rangle\right]^{2} .
\end{aligned}
$$


The first few expressions in (20) suggest that terms with one mean value of $z$ 's sum up to $\langle\ln [1+(A-1) z /(A+1)]\rangle$ for $\lambda=1$, but that there are also extra terms with two, three, ... mean values of $z$ 's. We now prove this statement.

From (18) with $\lambda$ set equal to one and $B_{n}+C_{n}+D_{n}+E_{n}+\ldots=X_{n}$

$$
\begin{aligned}
\ln \left(1+\frac{A^{2}}{A^{2}-1}\right. & \left.\left(\mathrm{e}^{X_{n}}-1\right)\right)=\ln \left(1+z_{n} \frac{A-1}{A+1}-\frac{\mathrm{e}^{-X_{n-1}-1}}{A^{2}-1}\right) \\
= & \ln \left(1+\frac{A-1}{A+1} z_{n}\right)+\ln \left(1-\frac{\mathrm{e}^{-X_{n-i}-1}}{A^{2}-1}\right) \\
& -\sum_{p=2}^{\infty} \frac{(-1)^{p}}{p} \sum_{r=1}^{p-1}\left(\begin{array}{l}
p \\
r
\end{array}\right)\left(\frac{A+1}{A+1} z_{n}\right)^{r}\left(\frac{1-\mathrm{e}^{-X_{n-1}}}{A^{2}-1}\right)^{p-r} .
\end{aligned}
$$

The second term on the right-hand side is

$$
\ln \left(1-\frac{\mathrm{e}^{-X_{n-1}-1}}{A^{2}-1}\right)=-X_{n-1}+\ln \left(1+\frac{A^{2}\left(\mathrm{e}^{X_{n-1}-1}\right)}{A^{2}-1}\right) .
$$

Taking averages and recalling that $z_{n}$ is independent of $x_{n-1}$, we obtain $\gamma(\omega)=\ln A+\langle X\rangle$

$$
\begin{aligned}
= & \ln A+\left\langle\ln \left(1+\frac{A-1}{A+1} z\right)\right\rangle \\
& -\sum_{r=1}^{\infty} \sum_{q=1}^{\infty}(-1)^{r+q}(r+q-1) ! \frac{1}{r !}\left\langle\left(\frac{A-1}{A+1} z\right)^{r}\right\rangle \frac{1}{q !}\left\langle\left(\frac{1-\mathrm{e}^{-x}}{A^{2}-1}\right)^{q}\right\rangle \\
= & \ln A+\left\langle\ln \left(1+\frac{A-1}{A+1} z\right)\right\rangle+\text { terms with at least two mean values. }
\end{aligned}
$$

From equations (19) we obtain the following expressions for averages of powers of $X$ up to order $\lambda^{4}$ :

$$
\begin{aligned}
& \langle X\rangle=-\frac{1}{2}\left\langle Z^{2}\right\rangle+\frac{1}{3}\left\langle Z^{3}\right\rangle-\frac{1}{4}\left\langle Z^{4}\right\rangle-\frac{1}{2}\left[\left(3+2 A^{2}\right) /\left(A^{4}-1\right)\right]\left(\left\langle Z^{2}\right\rangle\right)^{2}+\ldots, \\
& \left\langle X^{2}\right\rangle=\frac{A^{2}-1}{A^{2}+1}\left\langle Z^{2}\right\rangle-\frac{A^{2}-1}{A^{2}+1} \frac{A^{4}+1}{A^{4}+A^{2}+1}\left\langle Z^{3}\right\rangle \\
& +\frac{1}{12} \frac{\left(A^{2}-1\right)\left(11 A^{8}-11 A^{6}+36 A^{4}-11 A^{2}+11\right)}{\left(A^{4}+A^{2}+1\right)\left(A^{6}+A^{4}+A^{2}+1\right)}\left\langle Z^{4}\right\rangle \\
& +\frac{1}{2} \frac{6 A^{10}+11 A^{8}+A^{6}+36 A^{4}-5 A^{2}+11}{\left(A^{2}+1\right)\left(A^{4}+A^{2}+1\right)\left(A^{6}+A^{4}+A^{2}+1\right)}\left(\left\langle Z^{2}\right\rangle\right)^{2}+\ldots, \\
& \left\langle X^{3}\right\rangle=\frac{\left(A^{2}-1\right)^{2}}{A^{4}+A^{2}+1}\left\langle Z^{3}\right\rangle-\frac{3}{2} \frac{\left(A^{2}-1\right)^{2}\left(A^{6}+1\right)}{\left(A^{4}+A^{2}+1\right)\left(A^{6}+A^{4}+A^{2}+1\right)}\left\langle Z^{4}\right\rangle \\
& +\frac{\left(A^{2}-1\right)\left(A^{8}+4 A^{6}+A^{4}+A^{2}+3\right)}{\left(A^{2}+1\right)\left(A^{4}+A^{2}+1\right)\left(A^{6}+A^{4}+A^{2}+1\right)}\left(\left\langle Z^{2}\right\rangle\right)^{2}+\ldots, \\
& \left\langle X^{4}\right\rangle=\frac{\left(A^{2}-1\right)^{3}}{A^{6}+A^{4}+A^{2}+1}\left\langle Z^{4}\right\rangle+\frac{6\left(A^{2}-1\right)^{2}}{\left(A^{2}+1\right)\left(A^{6}+A^{4}+A^{2}+1\right)}\left(\left\langle Z^{2}\right\rangle\right)^{2}+\ldots,
\end{aligned}
$$

where we have used the shorthand notation

$$
Z=(A-1) /(A+1) z \text {. }
$$


Let us prove in general that a term in $\left\langle X^{p}\right\rangle$ involving $r z$ 's and $k$ expectation values behaves at least as $(A-1)^{p+r-k}$ when $A \rightarrow 1$. Let $t\left\{p_{i}\right\}=\left\langle B^{p_{1}} C^{p_{2}} D^{p_{3}} \ldots\right\rangle$, where $\Sigma p_{1}=p$. Multiplying equations (19) as $(a)^{p_{1}}(b)^{p_{2}}(\mathrm{c})^{p_{3}} \ldots$ gives an expression from $t\left\{p_{i}\right\}$ of the form

$$
t\left\{p_{i}\right\}=\frac{1}{\left(A^{2 p}-1\right)} \sum_{p_{2}, p_{3}^{\prime} \ldots} \sum_{j=0}^{p_{1}} a_{1, p_{2}^{\prime}, p_{3}^{\prime} .} t\left\{p_{1}-j, p_{2}^{\prime}, p_{3}^{\prime} \ldots\right\}\left\langle z^{\prime}\right\rangle(A-1)^{2 \prime}
$$

where $a_{\lambda, p_{2}^{\prime}, p_{3}^{\prime} . .}$ are known coefficients. It is possible to arrange the order of the calculation of these terms $t$ so that $t\left\{p_{1}^{\prime}\right\}$ is calculated before $t\left\{p_{i}\right\}$ if for some $k \geqslant 1$, $p_{i}^{\prime}=p_{i}$ for $i>k$ and $p_{k}^{\prime}<p_{k}$. The above relation then expresses $t\left\{p_{i}\right\}$ as a function of terms $t\left\{p_{1}-j, p_{2}^{\prime}, p_{3}^{\prime}, \ldots\right\}$ which have already been calculated, and the required result may be proved inductively. For $j=0, p^{\prime}=p_{1}+\Sigma_{i \geqslant 2} p_{l}^{\prime}>p$ and for $j \neq 0, p^{\prime \prime}=$ $p_{1}+\Sigma_{l \geqslant 2} p_{\imath}^{\prime} \geqslant p$. Furthermore $t\{0,0,0, \ldots\}=\left\langle B^{0} C^{0} \ldots\right\rangle=1, r=p=0$ and the lowest power of $(A-1)$ is indeed zero. Let $p_{i}>0$ for at least one value of $i$. For $j=0$ a term in $t\left\{p_{1}, p_{2}^{\prime}, \ldots\right\}$ with $r z$ 's and $k$ expectation values has lowest power of $(A-1)$ greater than or equal to $p^{\prime}+r-k$ which is larger than or equal to $p+1+r-k$, since $p^{\prime}>p$. The prefactor $\left(A^{2 p}-1\right)^{-1}$ contains a factor $(A-1)^{-1}$, and so the corresponding lowest power is at least $p+r-k$. For $j \neq 0$ a term in $t\left\{p_{1}-j, p_{2}^{\prime}, \ldots\right\}$ with $(r-j) z$ 's and $(k-1)$ expectation values has a lowest power of $(A-1)$ at least equal to $\left(p^{\prime \prime}-j\right)+(r-j)-k-$ $1 \geqslant p+r-2 j-k+1$. Multiplying by $\left(A^{2 p}-1\right)^{-1}\left\langle z^{\prime}\right\rangle(A-1)^{2 \prime}$ gives a term with $k$ mean values and $r$ powers of $z$, the exponent of $(A-1)$ being now at least $p+r-k$. Our assertion is therefore proved, and at a given order in $z$ (or $\lambda$ ) the term with the highest number of expectation values (highest value of $k$ ) also has the smallest power of $(A-1)$.

From this remark it follows that we can figure out the leading behaviour in $(A-1)$ of the fifth- and sixth-order terms, denoted $\langle F\rangle$ and $\langle G\rangle$, using (22) and (23):

$$
\begin{aligned}
& \langle F\rangle=\frac{3}{A^{2}-1}\left\langle\left(\frac{A-1}{A+1} z\right)^{2}\right\rangle\left\langle\left(\frac{A-1}{A+1} z\right)^{3}\right\rangle+\ldots \\
& \langle G\rangle=-\frac{15}{2} \frac{1}{\left(A^{2}-1\right)^{2}}\left[\left\langle\left(\frac{A-1}{A+1} z\right)^{3}\right\rangle\right]^{2}+\ldots
\end{aligned}
$$

We now obtain the small $\omega$ behaviour of the index $\gamma(\omega)$ from the weak disorder expansion (17). From (14)

$$
A-1=\omega^{1 / 2}+\frac{1}{2} \omega+\frac{1}{8} \omega^{3 / 2}+O\left(\omega^{5 / 2}\right) \text {. }
$$

Therefore to order $\omega^{2}$

$$
\begin{aligned}
\gamma(\omega)=\omega^{1 / 2}-\frac{1}{8} \omega\left\langle z^{2}\right\rangle-\frac{1}{384} \omega^{3 / 2}\left[16-16\left\langle z^{3}\right\rangle+15\left(\left\langle z^{2}\right\rangle\right)^{2}\right] \\
+\frac{1}{512} \omega^{2}\left[16\left\langle z^{2}\right\rangle-8\left(z^{4}\right\rangle+24\left(\left\langle z^{2}\right\rangle\right)^{2}+24\left\langle z^{2}\right\rangle\left\langle z^{3}\right\rangle-15\left(\left\langle z^{2}\right\rangle\right)^{6}\right]+\mathrm{O}\left(\omega^{5 / 2}\right) .
\end{aligned}
$$

For the Poisson distribution $\left\langle z^{2}\right\rangle=1,\left\langle z^{3}\right\rangle=2,\left\langle z^{4}\right\rangle=9, \ldots$ and

$$
\gamma(\omega)=\omega^{1 / 2}-\frac{1}{8} \omega+\frac{1}{384} \omega^{3 / 2}+\frac{1}{512} \omega^{2}+\mathrm{O}\left(\omega^{5 / 2}\right) .
$$

Through analytic continuation

$$
\begin{aligned}
& \operatorname{Re} \gamma(-\Omega-\mathrm{i} \varepsilon)=\frac{1}{8} \Omega+\frac{1}{512} \Omega^{2}+\mathrm{O}\left(\Omega^{3}\right), \\
& \operatorname{Im} \gamma(-\Omega-\mathrm{i} \varepsilon)=\Omega^{1 / 2}-\frac{1}{384} \Omega^{3 / 2}+\mathrm{O}\left(\Omega^{5 / 2}\right), \\
& \rho(\Omega)=\left(2 \pi \Omega^{1 / 2}\right)^{-1}\left(1-\frac{1}{128} \Omega+\mathrm{O}\left(\Omega^{2}\right)\right),
\end{aligned}
$$


A comparison of (36) and (15) shows that the next to leading term in the spectral function for the random lattice is a factor of $-\frac{1}{16}$ smaller than for the regular case, and this is significantly closer to the continuum density.

The interpretation of (28), a new term, since for a regular lattice $\gamma(-\Omega-i \varepsilon)$ is purely imaginary on the spectrum, is that it corresponds to an inverse localisation length $L(\Omega)$

$$
L(\Omega)=8 / \Omega-\frac{1}{8}+\mathrm{O}(\Omega) .
$$

Bounded solutions of (3) must most likely decrease exponentially with distance. Since $L(\Omega)$ goes to infinity as $\Omega$ goes to zero, the localisation does not appear in the continuum limit. The behaviour at large distances of small $\Omega$ wavefunctions is, however, affected by disorder, which might seem to be a limitation for the use of a random lattice in the computation of particle spectra. These effects should carefully be studied in higher dimensions. The low-order terms in $L(\Omega)$ seem to imply, as we shall verify, that it decreases with increasing $\Omega$. As $L(\Omega)$ reaches values of order unity, we expect results on a random lattice to be significantly different from those on a regular one. At that point a weak disorder expansion becomes irrelevant. In $\S 4$ an alternative method valid for large frequency will be studied.

\section{Large frequency expansion}

At large values of the frequency the states are strongly localised. Correspondingly $\gamma(\omega)$ can be determined by considering only sites which are a few spacings apart. From (9), $R_{n}$ may be written as a continued fraction, the presence of a large $\omega$ in the denominators allowing a truncation to any desired order of accuracy,

$$
R_{n}=2+\omega l_{n}-\frac{1}{2+\omega l_{n-1}-\frac{1}{2+\omega l_{n-2} \cdots}}
$$

So

$$
\gamma(\omega)=\left\langle\left\{\ln (2+\omega l)+\lim _{n \rightarrow \infty} \ln \left[1-\frac{1}{\left(2+\omega l_{n}\right)\left(2+\omega l_{n-1}-\frac{1}{2+\omega l_{n-2} \ldots}\right)}\right]\right\}\right)
$$

For the Poisson distribution

$$
\begin{aligned}
\langle\ln (2+\omega l)\rangle= & \ln 2+\omega\left\langle(2+\omega l)^{-1}\right\rangle \\
= & \ln 2+\mathrm{e}^{2 / \omega}\left[\ln \frac{\omega}{2}-\gamma+\frac{2}{\omega}-\frac{1}{2(2 !)}\left\{\frac{2}{\omega}\right\}^{2}+\frac{1}{3(3 !)}\left(\frac{2}{\omega}\right)^{2} \ldots\right], \\
& \left\langle\left((2+\omega l)^{-1}\right)^{p+1}\right\rangle=\left(p 2^{p} \omega\right)^{-1}+\mathrm{O}\left(\omega^{-2}\right), \quad p \geqslant 1 .
\end{aligned}
$$

Here $\gamma$ is Euler's constant 0.57722 and we shall use $C=\mathrm{e}^{\gamma}=1.78107$.

Then to order $\omega^{-2}$

$$
\gamma(\omega)=\ln 2+\omega\left\langle\frac{1}{2+\omega l}\right\rangle-\sum_{p=1}^{\infty} \frac{1}{p}\left[\left\langle\left(\frac{1}{2+\omega l}\right)^{p}\right\rangle\right]^{2}-\left[\left\langle\frac{1}{2+\omega l}\right\rangle\right]^{2}\left[\left\langle\left(\frac{1}{2+\omega l}\right)^{2}\right\rangle\right] .
$$

The last term is the first to involve $l_{n}, l_{n-1}$ and $l_{n-2}$. From (34), it is of order $(\ln \omega)^{2} / \omega^{3}$, and so can be dropped in a calculation up to $\omega^{-2}$. 
To this order

$\gamma(\omega)=\ln \frac{\omega}{C}+\frac{2}{\omega}\left(\ln \frac{\omega}{2 C}+1\right)-\frac{1}{\omega^{2}}\left[\left(\ln \frac{\omega}{2 C}-1\right\}^{2}+\eta-4\right]+\mathrm{O}\left[\left(\frac{\ln \omega}{\omega}\right)^{3}\right]$.

The constant $\eta$ is given by

$$
\eta=\sum_{1}^{\infty} \frac{1}{(t+1)^{2} t^{2} 4^{t}}=\operatorname{Li}_{2}\left(\frac{1}{4}\right)-1-3 \ln \frac{3}{4}=0.13070 .
$$

According to (36)

$\operatorname{Re} \gamma(-\Omega-i \varepsilon)$

$$
\begin{gathered}
=L^{-1}(\Omega)=\ln \frac{\Omega}{C}-\frac{2}{\Omega}\left(\ln \frac{\Omega}{2 C}+1\right)-\frac{1}{\Omega^{2}}\left[\left(\ln \frac{\Omega}{2 C}-1\right)^{2}+\eta-4-\pi^{2}\right],+\ldots, \\
\operatorname{Im} \gamma(-\Omega-\mathrm{i} \varepsilon)=\pi\left[1-2 / \Omega-\left(2 / \Omega^{2}\right)(\ln (\Omega / 2 C)-1)+\ldots\right] .
\end{gathered}
$$

For the spectral density this yields

$$
\rho(\Omega)=2 / \Omega^{2}+\left(4 / \Omega^{3}\right)\left(\ln (\Omega / 2 C)-\frac{3}{2}\right)+\ldots
$$

It is remarkable that logarithms enter these expressions; they arise from the nonvanishing probability of small intervals. We note that from $L^{-1}(\Omega)=\operatorname{Re} \gamma(-\Omega-i \varepsilon)$, $L(\Omega) \sim 1 / \ln \Omega$, implying strong localisation at large $\Omega$, while the density of states vanishes like $1 / \Omega^{2}$. In $d$ dimensions one conjectures that this behaviour generalises to $1 / \Omega^{d+1}$.

\section{Comparison with numerical simulations}

The smooth behaviour predicted at small and large values of $\Omega$ could leave the possibility of some accident or bump at intermediate values. This is what prompted a numerical simulation using the recurrence relation (9) directly for $\omega=-\Omega-i \varepsilon, \Omega>0$. The small $\varepsilon$ was chosen at first as 0.02 , then dropped to zero to count the number of sign changes in $R_{n}$ which give a direct measurement of the density $\rho(\Omega)$. Runs were performed with 250000 iterations and two random number generators were seen to yield compatible results. The data collected are shown in figure $1(a)$ (density $\rho), 1(b)(\operatorname{Im} \gamma$, integrated density), $1(c)$ ( $\operatorname{Re} \gamma$, inverse localisation length). The results show a smooth transition between the small and large values of $\Omega$ and are compared with the respective expansions. The agreement is quite reasonable. Finally in figure 2 we compare the density of states for a regular lattice, a random Poissonian one and the continuum.

The one-dimensional problem studied here allows not only detailed analysis, but also quite a straightforward numerical simulation. The situation unfortunately is not so simple in higher dimensions.

\section{Analytical study}

It is possible to investigate the probability density $P(R)$ of $(12)$ in greater detail. The equation involves the contractive map $R^{\prime} \rightarrow R=2-1 / R^{\prime}$ under which the interval $[1, \infty]$ is successively mapped onto $[1,1+1 / n]$. From $(11), P(R)$ is a distinct analytic 

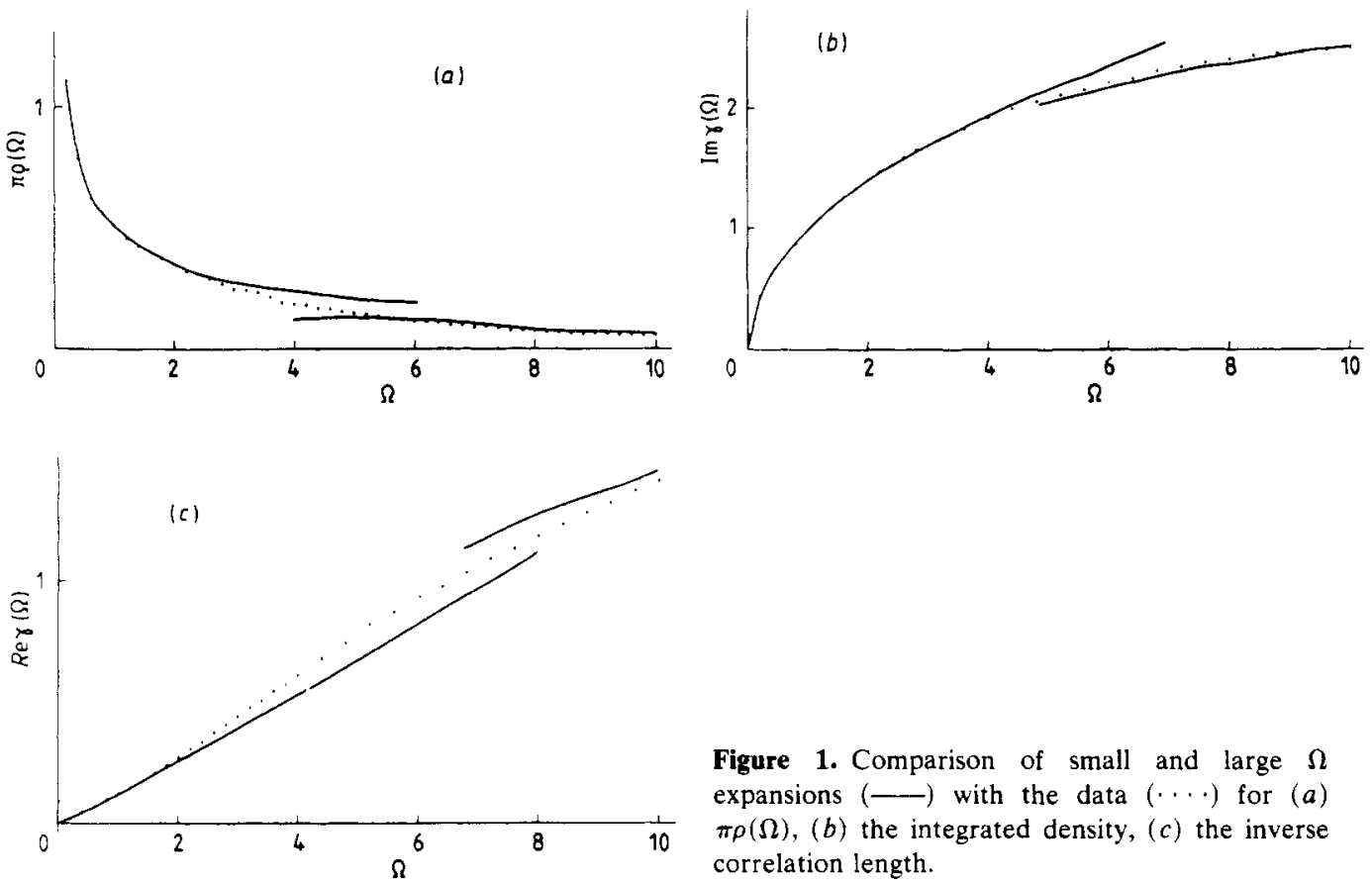

Figure 1. Comparison of small and large $\Omega$ expansions $(\longrightarrow)$ with the data $(\cdots)$ for $(a)$ $\pi \rho(\Omega),(b)$ the integrated density, $(c)$ the inverse correlation length.

function in each of the intervals $[1+1(n+1), 1+1 / n]$. It is therefore natural to set

$$
R=1+1 / x, \quad P(R)=x^{2} p(x), \quad 0 \leqslant x<\infty .
$$

Then

$p(x)=\frac{1}{\omega x^{2}} \int_{0}^{\infty} \mathrm{d} x^{\prime} p\left(x^{\prime}\right) \exp \left[-\frac{1}{\omega}\left(\frac{1}{x}-\frac{1}{x+1}\right)\right] \theta\left(x^{\prime}-\sup (x-1,0)\right)$

and

$$
\int_{0}^{x} \mathrm{~d} x p(x)=1
$$

For $0 \leqslant x<1$ we have

$$
p(x)=p_{0}(x)=\left(\alpha \omega x^{2}\right)^{-1} \mathrm{e}^{-1 / \omega x}
$$

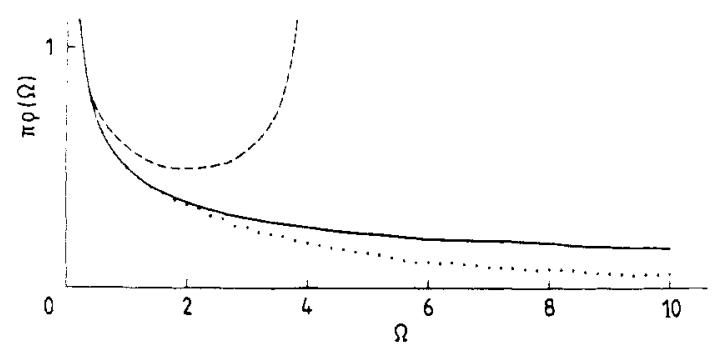

Figure 2. The density of states in the continuum (full line), on a regular lattice (broken line) and on a random lattice (dotted line). 
where $\alpha$ is a normalising constant such that

$$
\alpha^{-1}=\int_{0}^{\infty} \mathrm{d} x p(x) \exp [1 / \omega(x+1)] .
$$

To characterise the departure of $p(x)$ from $p_{0}(x)$ for $x>1$ we define $q(x)$ such that

$$
p(x)=p_{0}(x) q(x)
$$

It has the following properties.

$$
q(x)=1, \quad 0 \leqslant x \leqslant 1 .
$$

(ii) From (42) in differential form

$$
\exp [-1 / \omega(x+1)] \mathrm{d} q(x+1)+q(x) \mathrm{d}[\exp (-1 / \omega x)]=0
$$

and so $q(x)$ can be constructed recursively in the interval $[n, n+1]$ from its values in $[0, n]$, and using also $(45)$

$$
q(x+1)=1-\int_{0}^{x} \mathrm{~d} y q(y) \frac{1}{\omega y^{2}} \exp \left[-\frac{1}{\omega}\left(\frac{1}{y}-\frac{1}{y+1}\right)\right] .
$$

(iii) The function $q(x)$ is positive and monotonically decreasing as a consequence of (47). It decreases at least as $\omega^{1-x} / \Gamma(x)$ for large $x$. It is analytic in each interval $(n, n+1)$ an is globally infinitely differentiable.

(iv) From (43)

$$
\alpha(\omega)=\int_{0}^{\infty} \mathrm{d} x q(x) \frac{\mathrm{e}^{-1 / \omega x}}{\omega x^{2}}=\sum_{1}^{\infty} q(n) \mathrm{e}^{-1 / n \omega} .
$$

The second expression may be obtained from (48) using

$$
\int_{0}^{\infty} \mathrm{d} x q(x) \frac{1}{\omega x^{2}} \mathrm{e}^{-1 / \omega x}=\int_{0}^{1} \mathrm{~d} x \frac{\mathrm{e}^{-1 / \omega x}}{\omega x^{2}}+\int_{1}^{\infty} \mathrm{d} x q(x) \frac{\mathrm{e}^{-1 / \omega x}}{\omega x^{2}} .
$$

Repeating the process yields (50).

(v) The index $y$ is then given by

$$
\alpha(\omega) \gamma(\omega)=\int_{0}^{\infty} \mathrm{d} x q(x) \frac{\mathrm{e}^{-1 / \omega x}}{x}=\int_{0}^{1} \frac{\mathrm{d} x}{x} \mathrm{e}^{-1 / \omega x}+\sum_{2}^{\infty} q(n) \omega s_{n-2}(\omega) \mathrm{e}^{-1 / n \omega}
$$

where $s_{p}(\omega) \equiv s_{p}(\omega, x=1)$ and $s_{p}(\omega, x)$ is a polyomial in both $\omega$ and $x$ of degree $p$ in $\omega$ with

$$
\begin{aligned}
& s_{0}(\omega, x)=x, \\
& s_{p}(\omega, x)=\omega\left(x+p^{2}\right)(\mathrm{d} / \mathrm{d} x) s_{p-1}(\omega, x)+s_{p-1}(\omega, x), \\
& s_{p}(\omega)=s_{p}(\omega, 1) .
\end{aligned}
$$

To get the first equality in (52) we proceed as follows:

$$
\begin{aligned}
\alpha(\omega) \gamma(\omega) & =\int_{0}^{\infty} \mathrm{d} x q(x) \frac{1}{\omega x^{2}} \mathrm{e}^{-1 / \omega x} \ln \frac{1+x}{x} \\
& =-\int_{0}^{\infty} \mathrm{d} x \mathrm{e}^{-1 / \omega x}\left[\frac{\mathrm{d} q(x)}{\mathrm{d} x} \ln \frac{1+x}{x}+q(x)\left(\frac{1}{1+x}-\frac{1}{x}\right)\right] \\
& =\int_{0}^{\infty} \mathrm{d} x q(x) \mathrm{e}^{-1 / \omega x} \ln \frac{2+x}{1+x}+\int_{0}^{\infty} \mathrm{d} x \mathrm{e}^{-1 / \omega x} q(x)\left(\frac{1}{x}-\frac{1}{x+1}\right)
\end{aligned}
$$


where the last step follows from (48). Iterating the process $n$ times and taking the limit $n \rightarrow \infty$ gives the first equality in (52). To obtain the seond we write

$$
\begin{aligned}
\int_{0}^{\infty} \mathrm{d} x q(x) & \frac{\mathrm{e}^{-1 / \omega x}}{x}=\int_{0}^{1} \frac{\mathrm{d} x}{x} \mathrm{e}^{-1 / \omega x}-\omega \int_{1}^{\infty} \mathrm{d} x \mathrm{e}^{-1 / \omega(x+1)} x \frac{\mathrm{d}}{\mathrm{d} x} q(x+1) \\
& =\int_{0}^{1} \frac{\mathrm{d} x}{x} \mathrm{e}^{-1 / \omega x}+\omega q(2) \mathrm{e}^{-1 / 2 \omega}+\omega \int_{2}^{\infty} \mathrm{d} x q(x) \frac{\omega x^{2}+x-1}{\omega x^{2}} \mathrm{e}^{-1 / \omega x} .
\end{aligned}
$$

From (48) the last integral may be written

$$
\omega(1+4 \omega) q(3) \mathrm{e}^{-1 / 3 \omega}+\int_{3}^{\infty} \mathrm{d} x q(x) \frac{\mathrm{d}}{\mathrm{d} x}\left\{\left[\omega(x-1)^{2}+x-2\right] \mathrm{e}^{-1 / \omega x}\right\} .
$$

Repeating this process gives the second equality in (52) as well as the recurence relations (53) which yield

$$
\begin{aligned}
& s_{0}(\omega)=1, \quad s_{1}(\omega)=(1+4 \omega), \quad s_{2}(\omega)=(1+4 \omega)(1+9 \omega), \\
& s_{3}(\omega)=(1+4 \omega)(1+9 \omega)+16 \omega\left[18 \omega^{2}+(1+4 \omega)(1+6 \omega)\right] .
\end{aligned}
$$

From (51) and (52), $\gamma(\omega)$ is the ratio of two series, each involving $q$ evaluated at integer points. The large frequency expansion of $\S 4$ can be reconstructed from these expressions.

\section{The replica method}

It is of interest to calculate two- and higher-point Green functions in order to learn something about the behaviour of states in space. We shall illustrate these computations using the replica method. The latter reduces a quenched average to an annealed one, the price paid being the introduction of $n$ replicas of the partition function in the limit $n \rightarrow 0$. We first describe briefly the technique, show its relation with our previous analysis and prove that it yields for the exponent $\gamma(\omega)$ the same results as before. The low frequency expansion (26) is explicitly recovered in agreement with Stephen and Kariotis (1982). We then extend the method to the calculation of Green functions at non-zero separations.

\subsection{Calculation of $\gamma(\omega)$}

We work in terms of the function $\varphi_{n} \equiv \varphi\left(x_{n}\right)$ introduced in $\S 2$ and study the partition function of a statistical model corresponding to (5). In the presence of a source $J$, the free energy is

$$
F(J)=\left\langle\ln \int \prod_{i} \mathrm{~d} \varphi_{i} \exp \left[-\left(H(\varphi, l)+\sum_{i} J_{i} \varphi\right)\right]\right\rangle
$$

where

$$
H(\varphi, l)=\frac{1}{2} \sum_{i}\left[\left(\varphi_{i}-\varphi_{1+1}\right)^{2} / l_{l}+\omega \varphi_{i}^{2}\right]
$$

If $n$ replicas of the model are introduced (labelled by indices $\alpha$ ranging from 1 to $n$ ), $H$ will be replaced by $\Sigma_{\alpha} H\left(\varphi_{\alpha}, l\right)$ which from its quadratic nature will be equal to the same function involving vector valued $\varphi$ 's and $O(n)$-invariant scalar products. The 
free energy can then be written in terms of an $n \rightarrow 0$ limit as

$$
F(J)=\left\langle\lim _{n \rightarrow 0} \frac{1}{n} \int \prod \mathrm{d} \boldsymbol{\varphi}_{1}\left\{\exp \left[-\left(H(\boldsymbol{\varphi}, l)+\sum \boldsymbol{J}_{i} \boldsymbol{\varphi}_{i}\right)\right]-1\right\}\right\rangle
$$

where $H(\varphi, l)$ is given by an expression similar to (56) with vector valued $\varphi$ 's instead of scalar ones. A calculation will be easily feasible if the limit $n \rightarrow 0$ and the average can be interchanged. This appears justified as we will show by computing $\gamma(\omega)$, related to the propagator at zero separation $G_{00}(\omega)$ through

$$
G_{00}(\omega)=\frac{\mathrm{d} \gamma(\omega)}{\mathrm{d} \omega}=\left.\frac{\partial^{2}}{\partial J_{0}^{2}} F(J)\right|_{J=0}=\lim _{n \rightarrow 0} \int \mathrm{d} \varphi \frac{\varphi^{2}}{n} \exp \left(-\omega \varphi^{2} / 2\right) \psi^{2}(\varphi)
$$

Here $\psi$ is the contribution to the right or to the left of the origin in the path integral, and represents the ground state wavefunction of the transfer matrix. Henceforth the limit $n \rightarrow 0$ is always understood. From translational invariance $\psi$ satisfies

$$
\psi\left(\varphi^{2}\right)=\int \mathrm{d} \varphi^{\prime}\left\langle\exp \left[-(2 l)^{-1}\left(\varphi-\varphi^{\prime}\right)^{2}\right]\right\rangle \exp \left(-\omega \varphi^{\prime 2} / 2\right) \psi\left(\varphi^{\prime 2}\right)
$$

We have taken into account the fact that the ground state is $O(n)$ invariant. Due to the limit $n \rightarrow 0$ the corresponding eigenvalue is 1 . In the absence of disorder (59) would reduce to

$$
\begin{aligned}
& \psi_{0}\left(\varphi^{2}\right)=\int \mathrm{d} \boldsymbol{\varphi}^{\prime} \exp \left[-\left(\varphi-\varphi^{\prime}\right)^{2} / 2-\omega \boldsymbol{\varphi}^{\prime 2} / 2\right] \psi_{0}\left(\boldsymbol{\varphi}^{\prime 2}\right), \\
& \psi_{0}\left(\boldsymbol{\varphi}^{2}\right)=\exp \left[-\frac{1}{2} \alpha(\omega) \varphi^{2}\right], \quad \alpha(\omega)=\left(\omega+\omega^{2} / 4\right)^{1 / 2}-\omega / 2 .
\end{aligned}
$$

If we note that

$$
\lim _{n \rightarrow 0} \int \mathrm{d} \varphi \exp \left(-\beta \varphi^{2}\right) n^{-1}\left(\varphi^{2}\right)^{1+t}=t ! / 2 \beta^{t+1}
$$

we recover the known result

$$
\left.G_{00}(\omega)\right|_{0}=\frac{\mathrm{d} \omega_{0}(\omega)}{\mathrm{d} \omega}\left[2\left(\omega+\omega^{2} / 4\right)^{1 / 2}\right]^{-1}=\frac{\mathrm{d}}{\mathrm{d} \omega} \ln A
$$

with the same $A$ introduced in (4), i.e. $A=1+\omega / 2+\left(\omega+\omega^{2} / 4\right)^{1 / 2}$. One way to proceed next is to perform the weak disorder (small $\lambda$ ) expansion analogous to the one studied in $\S 3$. To demonstrate its working we shall simply compute the $\lambda^{2}$ corrections (the first non-vanishing one). Detailed calculations of similar nature are reported by Stephen and Kariotis (1982) using a saddle point method. We set $\psi\left(\varphi^{2}\right)=$ $\psi_{0}\left(\varphi^{2}\right)\left[1+\psi_{2}\left(\varphi^{2}\right)+\ldots\right]$. Similarly $l=1+z$ and we keep terms up to order $z^{2}$. This yields

$$
\psi_{2}\left(\varphi^{2}\right)=\int \mathrm{d} \varphi^{\prime} \exp \left[-\frac{A}{2}\left(\varphi^{\prime}-\frac{\varphi}{A}\right)^{2}\right]\left[\psi_{2}\left(\varphi^{2}\right)+\frac{1}{8}\left\langle z^{2}\right)\left[-4\left(\varphi^{\prime}-\varphi\right)^{2}+\left[\left(\varphi^{\prime}-\varphi\right)^{2}\right]^{2}\right] .\right.
$$

It is readily verified that

$$
\begin{aligned}
\int \mathrm{d} \varphi^{\prime} \exp [ & \left.-\frac{A}{2}\left(\varphi^{\prime}-\frac{\varphi}{A}\right)^{2}\right]\left\{1, \varphi^{\prime 2},\left(\varphi^{\prime 2}\right)^{2},\left(\varphi^{\prime}-\varphi\right)^{2},\left[\left(\varphi^{\prime}-\varphi\right)^{2}\right]^{2}\right\} \\
& =\left\{1, \frac{\varphi^{2}}{A^{2}}, \frac{\left(\varphi^{2}\right)^{2}}{A^{4}}+\frac{4\left(\varphi^{2}\right)}{A^{3}},\left(\frac{A-1}{A}\right)^{2} \varphi^{2},\left(\frac{A-1}{A}\right)^{4}\left(\varphi^{2}\right)^{2}+\frac{4}{A}\left(\frac{A-1}{A}\right)^{2} \varphi^{2}\right\}
\end{aligned}
$$


which is obtained by studying the general transform of $\exp \left[b \varphi^{\prime 2}+c\left(\varphi^{\prime}-\varphi\right)^{2}\right]$. This shows that $\psi_{2}\left(\varphi^{2}\right)$ is a second-degree polynomial in $\varphi^{2}$, which is found equal to

$$
\psi_{2}\left(\varphi^{2}\right)=-\frac{\left\langle z^{2}\right\rangle}{8}\left(\frac{4(A-1)^{3}\left(A^{3}-1\right)}{\left(A^{2}-1\right)\left(A^{4}-1\right)} \varphi^{2}-\frac{(A-1)^{4}}{A^{4}-1}\left(\varphi^{2}\right)^{2}\right) .
$$

Inserting in equation (58) yields, with $\beta=\left(\omega+\omega^{2} / 4\right)^{1 / 2}=\left(A^{2}-1\right) / 2 A$,

$$
\begin{aligned}
\left.G_{00}(\omega)\right|_{2}= & \int \mathrm{d} \varphi \exp \left(-\beta \varphi^{2}\right) \frac{\varphi^{2}}{n} 2 \psi_{2}\left(\varphi^{2}\right) \\
& =-\frac{\left\langle z^{2}\right\rangle}{8} \frac{1}{\beta^{2}}\left(\frac{4(A-1)^{3}\left(A^{3}-1\right)}{\left(A^{2}-1\right)\left(A^{4}-1\right)}-\frac{2}{\beta} \frac{(A-1)^{4}}{A^{4}-1}\right) \\
& =-2\left\langle z^{2}\right\rangle \frac{A^{2}}{(A+1)^{4}}=\frac{\mathrm{d}}{\mathrm{d} \omega}\left[-\frac{\left\langle z^{2}\right\rangle}{2}\left(\frac{A-1}{A+1}\right)^{2}\right] \underset{\omega \rightarrow 0}{\sim}-\frac{\left\langle z^{2}\right\rangle}{8}+\frac{\omega}{16}\left\langle z^{2}\right\rangle+\ldots
\end{aligned}
$$

and this agrees of course with our previous result, equations (20) and (26). The amount of algebra required here is far greater than the direct computation of $\S 3$. Of course one could proceed similarly to compute higher terms, the correction terms $\psi_{3}, \psi_{4} \ldots$ being polynomials in $\varphi^{2}$.

We may also define a ground state wavefunction for the variables $Q_{i}=\left(\varphi_{i+1}-\varphi_{i}\right) / l_{i}$ with corresponding Hamiltonian $H=\Sigma_{i} \frac{1}{2}\left(Q_{i}-Q_{i+1}\right)^{2}+\frac{1}{2} \omega l_{i} Q_{i}^{2}$. Generalising to $n$ components and letting $n \rightarrow 0$, the wavefunction $\chi\left(\boldsymbol{Q}^{2}\right)$ satisfies

$$
\chi\left(\boldsymbol{Q}^{2}\right)=\int \mathrm{d} \boldsymbol{Q}^{\prime} \exp \left[-\frac{1}{2}\left(\boldsymbol{Q}-\boldsymbol{Q}^{\prime}\right)^{2}\right]\left\langle\exp \left(-\frac{1}{2} \omega l \boldsymbol{Q}^{\prime 2}\right)\right\rangle \chi\left(\boldsymbol{Q}^{\prime 2}\right) .
$$

The relation between $\chi$ and $\psi$ is a Fourier transform (Stephen and Kariotis 1982)

$$
\begin{aligned}
& \psi(\boldsymbol{\varphi})=\int \mathrm{d} \boldsymbol{Q} \exp (\mathrm{i} \boldsymbol{\varphi} \cdot \boldsymbol{Q} \sqrt{\omega})\left\langle\exp \left(-\frac{1}{2} \omega l \boldsymbol{Q}^{2}\right)\right\rangle \chi(\boldsymbol{Q}) \\
& \left\langle\exp \left(-\frac{1}{2} \omega l \boldsymbol{Q}^{2}\right)\right\rangle_{\chi}(\boldsymbol{Q})=\int \mathrm{d} \boldsymbol{\varphi} \exp (-\mathrm{i} \sqrt{\omega} \boldsymbol{\varphi} \cdot \boldsymbol{Q}) \psi(\boldsymbol{\varphi})
\end{aligned}
$$

The validity of the replica method can be established (Lin 1970) by studying the relation of $\psi$ and $\varphi$ with the probability $P(R)$ of $\S 2$ or alternatively $p(x)$ of $(44)$. We have

$$
\begin{gathered}
\chi\left(\boldsymbol{Q}^{2}\right)\left\langle\exp \left(-\frac{1}{2} \omega l \boldsymbol{Q}^{2}\right)\right\rangle=\frac{1}{2} \int_{1}^{\infty} \mathrm{d} R P(R) \exp \left(-\frac{R-1}{2} \boldsymbol{Q}^{2}\right) \\
P(R)=\frac{1}{2 \pi \mathrm{i}} \int_{c-\mathrm{i} \infty}^{c+i \infty} \mathrm{d} \boldsymbol{Q}^{2} \exp \left(\frac{R-1}{2} \boldsymbol{Q}^{2}\right) \chi\left(\boldsymbol{Q}^{2}\right)\left\langle\exp \left(-\frac{1}{2} \omega l \boldsymbol{Q}^{2}\right)\right\rangle \\
\psi\left(\boldsymbol{\varphi}^{2}\right)=\frac{1}{2} \int_{1}^{\infty} \mathrm{d} R \exp \left(-\frac{\omega}{2} \frac{\varphi^{2}}{R-1}\right) P(R)=\frac{1}{2} \int_{0}^{\infty} \mathrm{d} x p(x) \exp \left(-\frac{1}{2} \omega x \boldsymbol{\varphi}^{2}\right) \\
p(x)=\omega \frac{1}{2 \pi \mathrm{i}} \int_{c-\mathrm{i} \infty}^{c+\mathrm{i} \infty} \mathrm{d} \boldsymbol{\varphi}^{2} \exp \left(\frac{1}{2} \omega x \varphi^{2}\right) \psi\left(\varphi^{2}\right)
\end{gathered}
$$

The Laplace transform relations (67) (and (68)) imply that $(R-1) / 2$ and $\boldsymbol{Q}^{2}$ (and $\frac{1}{2} \omega x$ and $\left.\varphi^{2}\right)$ are conjugate variables. Similar relations hold in a variety of one- 
dimensional problems. From (68), we derive

$$
G_{00}(\omega)=\frac{\mathrm{d} \gamma(\omega)}{\mathrm{d} \omega}=\int_{0}^{\infty} \mathrm{d} x_{1} \int_{0}^{\infty} \mathrm{d} x_{2} \frac{p\left(x_{1}\right) p\left(x_{2}\right)}{4 \omega\left(1+x_{1}+x_{2}\right)} .
$$

\subsection{Green functions at arbitrary separation}

The field amplitude at a particular point in space is not defined unless it happens to belong to a given random lattice. Correspondingly there is some arbitrariness in the definition of Green functions at non-vanishing separations. We choose to take averages over lattices which contain all points at which the amplitude is required. For non-zero separations the number of spacings between distinct points can therefore take all values larger than or equal to one. For the two-point function evaluated between 0 and $L$ we get the expression

$$
G_{O L}(\omega)=\frac{\sum_{p=1}^{\infty} \int \Pi \mathrm{d} l_{i} \int \Pi \mathrm{d} \varphi_{i} \exp \left[-H(\varphi, l)-\Sigma l_{i}\right]\left[\left(\varphi_{0} \cdot \varphi_{L}\right) / n\right] \delta\left(L-\Sigma_{0}^{p-1} l_{i}\right)}{\Sigma_{p=1}^{\infty} \int \Pi \mathrm{d} l_{i} \exp \left(-\Sigma l_{i}\right) \delta\left(L-\Sigma_{0}^{p-1} l_{i}\right)} .
$$

The denominator is equal to one for the Poisson distribution

$$
\sum_{p=1}^{\infty} \int \Pi \mathrm{d} l_{i} \exp \left(-\sum l_{i}\right) \delta\left(L-\sum_{0}^{p-1} l_{i}\right)=\mathrm{e}^{-L} \sum_{p=1}^{\infty} \frac{L^{p-1}}{(p-1) !}=1 .
$$

In the numerator it is understood that $x_{0}=0, x_{1}=l_{0} \ldots x_{L}=\Sigma_{0}^{p-1} l_{1}$ and $\varphi_{L}$ stands in fact in our former notation for $\varphi_{p}$. After averages $G_{0 L}(\omega)$ is extended as a symmetric function of $L$ and we can write

$$
\begin{aligned}
G_{0 L}(\omega)=G_{0,-L}(\omega)=\int \mathrm{d} \boldsymbol{\varphi} \mathrm{d} \boldsymbol{\varphi}^{\prime} \psi\left(\boldsymbol{\varphi}^{\prime}\right) \frac{\boldsymbol{\varphi}^{\prime} \cdot \boldsymbol{\varphi}}{n} \psi(\boldsymbol{\varphi}) \exp \left(-\frac{1}{2} \omega \boldsymbol{\varphi}^{\prime 2}\right) F\left(\boldsymbol{\varphi}^{\prime}, \boldsymbol{\varphi}, L\right), \\
F\left(\boldsymbol{\varphi}^{\prime}, \boldsymbol{\varphi} ; L\right)=\sum_{p=1}^{\infty} \int \prod_{i=0}^{p=1} \mathrm{~d} l_{l} \exp \left(-l_{l}\right) \prod_{1}^{p-1} \mathrm{~d} \boldsymbol{\varphi}_{i} \\
\quad \times \exp \left[-\frac{1}{2} \sum_{i=0}^{p-1}\left(\frac{1}{l_{l}}\left(\boldsymbol{\varphi}_{i+1}-\boldsymbol{\varphi}_{i}\right)^{2}+\omega \boldsymbol{\varphi}_{l}^{2}\right)\right] \delta\left(L-\sum_{0}^{p-1} l_{i}\right) .
\end{aligned}
$$

For $p=1$ there is no $\varphi$ integral in $F$. Using the symmetry $L \rightarrow-L$ we can Fourier transform $G$ and $F$ as

$\tilde{G}_{k}(\omega)=\int \mathrm{d} L \mathrm{e}^{-1 L k} G_{0 L}(\omega)=\int \mathrm{d} \boldsymbol{\varphi} \mathrm{d} \boldsymbol{\varphi}^{\prime} \psi\left(\boldsymbol{\varphi}^{\prime}\right) \frac{\boldsymbol{\varphi}^{\prime} \cdot \boldsymbol{\varphi}}{n} \psi(\boldsymbol{\varphi}) \exp \left(-\frac{1}{2} \boldsymbol{\varphi}^{\prime 2}\right) \tilde{F}\left(\boldsymbol{\varphi}^{\prime}, \boldsymbol{\varphi} ; k\right)$,

$\tilde{F}\left(\boldsymbol{\varphi}^{\prime}, \boldsymbol{\varphi}, k\right)=\sum_{p=1}^{\infty}\left(M^{p}\left(\boldsymbol{\varphi}^{\prime}, \boldsymbol{\varphi} ; k\right)+k \rightarrow-k\right)$.

Here $M$ is an operator with kernel

$$
M\left(\boldsymbol{\varphi}^{\prime}, \boldsymbol{\varphi}\right)=\left\langle\exp \left[-\mathrm{i} k l-\frac{1}{2}\left(\boldsymbol{\varphi}^{\prime}-\boldsymbol{\varphi}\right)^{2} / l\right]\right\rangle \exp \left(-\frac{1}{2} \omega \boldsymbol{\varphi}^{2}\right) .
$$

and $M^{P}$ is its pth power. Because of $\mathrm{O}(n)$ invariance the eigenstates of $M$ transform as spherical harmonics. Since $\psi$ is a scalar it follows from (72) that we need only compute vector harmonics of the form $\varphi \theta_{r}\left(\varphi^{2}\right)$, the index $r$ being used to distinguish among several possible solutions. 
For the Poisson distribution we can write

$\left(\mu_{r}(k, \omega)\right)^{-1} \varphi^{\prime} \theta_{r}\left(\varphi^{\prime 2}\right)=\int \mathrm{d} \varphi\left\langle\exp \left[-\frac{1}{2}\left(\varphi^{\prime}-\varphi\right)^{2}(1+\mathrm{i} k) / l\right]\right\rangle \exp \left(-\frac{1}{2} \omega \varphi^{2}\right) \varphi \theta_{r}\left(\varphi^{2}\right)$

We use the following normalisations, compatible with our previous study

$$
\int \mathrm{d} \varphi \psi^{2}\left(\varphi^{2}\right) \exp \left(-\frac{1}{2} \omega \varphi^{2}\right)=\int \mathrm{d} \varphi\left(\varphi^{2} / n\right) \theta_{r}^{2}\left(\varphi^{2}\right) \exp \left(-\frac{1}{2} \omega \varphi^{2}\right)=1
$$

and get the following expression for the Fourier transform of the propagator

$$
\begin{aligned}
& \tilde{G}_{k}(\omega)=\sum_{r}\left(\frac{A_{r}^{2}(k, \omega)}{(1+\mathrm{i} k) \mu_{r}(k, \omega)-1}+k \rightarrow-k\right), \\
& A_{r}(k, \omega)=\int \mathrm{d} \boldsymbol{\varphi}\left(\varphi^{2} / n\right) \psi\left(\varphi^{2}\right) \theta_{r}\left(\varphi^{2}\right) \exp \left(-\frac{1}{2} \omega \varphi^{2}\right) .
\end{aligned}
$$

It can be remarked that the algebra is such that $G_{0 L}$ as defined here does not reduce to $G_{00}$ defined previously, in the limit $L \rightarrow 0$. This is a drawback of our method due to the fact that randomness affects the kinetic rather than the potential term in $H$. Indeed it can be checked that in the limit $L \rightarrow 0$ the only nonvanishing contribution arises from the one separation term in (70) with a result

$$
\lim _{L \rightarrow 0} G_{0 L}(\omega)=\int \mathrm{d} \varphi \psi^{2}\left(\varphi^{2}\right)\left(\varphi^{2} / n\right) \exp \left(-\omega \varphi^{2}\right)
$$

with a weight $\exp \left(-\omega \varphi^{2}\right)$ instead of $\exp \left(-\frac{1}{2} \omega \varphi^{2}\right)$ in the correct answer (59). Hence one will rather study $G_{0 L}$ in the large separation limit where the poles in (76) will generate a sequence of exponential terms each with oscillatory and damped behaviour, the latter being due to localisation effects. To this end we have performed a small $\omega$ expansion of the various quantities involved to order $\omega$ (keeping in mind that $\varphi^{2}$ is of the order $1 / \sqrt{\omega})$. We set

$$
x=\varphi^{2}, \quad x_{k}=(\mathrm{i}+k) \varphi^{2}, \quad \omega_{k}=\omega /(1+\mathrm{i} k) .
$$

Our results are as follows:

$$
\begin{aligned}
& \psi(x)=\exp \left(-\frac{1}{2} \sqrt{\omega} x\right)\left[1+\left(\frac{1}{16} \omega-\omega^{3 / 2} / 3(16)^{2}\right) x+\left(\frac{1}{32} \omega^{3 / 2}-\left(6(16)^{2}\right)^{-1} \omega^{2}\right) x^{2}\right. \\
& \left.-\left(18(16)^{2}\right)^{-1} \omega^{5 / 2} x^{3}+\omega^{3} x^{4} / 8(16)^{2}+\mathrm{O}\left(\omega^{3 / 2}\right)\right] \text {, } \\
& \theta_{0}(x)=N_{0}^{-1} \exp \left(-\frac{1}{2} \sqrt{\omega_{k}} x_{k}\right)\left[1+\left(-\frac{1}{8} \omega_{k}+\frac{1}{128} \omega_{k}^{3 / 2}\right) x_{k}+\left(\frac{1}{32} \omega_{k}^{3 / 2}+\frac{11}{512} \omega_{k}^{2}\right) x_{k}^{2}\right. \\
& \left.-(7 / 9.128) \omega_{k}^{5 / 2} x_{k}^{3}+\left(8(16)^{2}\right)^{-1} \omega_{k}^{3} x_{k}^{4}+\mathrm{O}\left(\omega^{3 / 2}\right)\right] \text {, } \\
& \left(\mu_{0}(\omega, k)\right)^{-1}=1-\omega_{k}^{1 / 2}+\frac{3}{4} \omega_{k}-\frac{31}{64} \omega_{k}^{3 / 2}+\mathrm{O}\left(\omega^{2}\right) \text {, } \\
& N_{0}^{-2}=2(1+i k) \omega_{k}^{1 / 2}\left[1+\frac{5}{8} \omega_{k}^{1 / 2}-\frac{7}{192} \omega_{k}+O\left(\omega^{3 / 2}\right)\right] \text {, } \\
& \theta_{1}(x)=\frac{1}{N_{1}} \exp \left(-\frac{1}{2} \sqrt{\omega_{k}} x_{k}\right)\left[1-\left(\omega_{k}^{1 / 2}+\frac{11}{8} \omega_{k}\right) x_{k}+\frac{17}{32} \omega_{k}^{3 / 2} x_{k}-\frac{1}{32} \omega_{k}^{2} x_{k}^{3}+\mathrm{O}(\omega)\right], \\
& \left(\mu_{1}(\omega, k)\right)^{-1}=1-3 \omega_{k}^{1 / 2}+\frac{25}{4} \omega_{k}+\mathrm{O}\left(\omega^{3 / 2}\right), \quad 1 / N^{2}=2(1+i k) \omega_{k}^{1 / 2}+\mathrm{O}(\omega) .
\end{aligned}
$$

In general $\mu_{r}\left(\omega_{k}\right) \sim 1+(2 r+1) \omega_{k}^{1 / 2}+\ldots, r=0,1,2, \ldots$ We can then obtain the 
numerators $A_{r}^{2}$ in (76). In particular there is a sum rule of the form

$$
G_{00}(\omega)=\mathrm{d} \gamma(\omega) / \mathrm{d} \omega=\sum_{r} A_{r}^{2}(\omega, 0)
$$

which can be used to check (79) and (80). Indeed

$$
\begin{aligned}
& A_{0}(\omega, 0)=\left(2 \omega N_{0}(\omega, 0)\right)^{-1}\left[1-\frac{7}{16} \omega^{1 / 2}+\frac{35}{192} \omega+\ldots\right], \\
& A_{1}(\omega, 0)=\left(N_{1}(\omega, 0)\right)^{-1}\left[-\frac{3}{32}+\ldots\right],
\end{aligned}
$$

which yields

$$
G_{00}(\omega)=A_{0}^{2}(\omega, 0)+A_{1}^{2}(\omega, 0)+\mathrm{O}(\omega)=\left(1 / 2 \omega^{1 / 2}\right)\left[1-\frac{1}{4} \omega^{1 / 2}+\frac{1}{128} \omega+\mathrm{O}\left(\omega^{3 / 2}\right)\right]
$$

in agreement with (27).

We can interpret the denominators in (76) as giving a set of dispersion relations when continued to $\omega=-\Omega-i \varepsilon$. As stated previously they will generate a large $L$ behaviour of $G_{0 L}(-\Omega-i \varepsilon)$ of the form

$$
\lim _{L \rightarrow \infty} G_{0 L}(-\Omega-\mathrm{i} \varepsilon) \sim \sum_{r} g_{r} \exp \left(\mathrm{i} \operatorname{Re} k_{r} L-\operatorname{Im} k_{r} L\right) .
$$

From $(80)$ we find for the first two terms

$$
\begin{array}{ll}
\operatorname{Re} k_{0}=\Omega^{1 / 2}\left[1+\frac{1}{64} \Omega+\mathrm{O}\left(\Omega^{2}\right)\right], & \operatorname{Im} k_{0}=\frac{1}{4} \Omega+\mathrm{O}\left(\Omega^{2}\right), \\
\operatorname{Re} k_{1}=3 \Omega^{1 / 2}[1+\mathrm{O}(\Omega)], & \operatorname{Im} k_{1}=\frac{7}{4} \Omega[1+\mathrm{O}(\Omega)] .
\end{array}
$$

The leading behaviour has an exponential decay in $\mathrm{e}^{-(\Omega / 4) L}$. This result may also be derived from the appendix of Casher and Lebowitz (1971). Our naive expectations were that it behaved like a wavefunction, i.e. in $\exp [-L / L(\Omega)]$, where $L(\Omega)$ is the localisation length $L(\Omega) \simeq \Omega / 8$. The factor 2 of disagreement can be attributed to the different averages involved.

Of course higher Green function can also be computed. Although we are considering a free field theory, there are effective interactions between 'particles' generated by the average over randomness. The second dispersion relation in (85) shows that the first excited state behaves like a set of three free particles, but that it is more localised. To understand such effects it is easier to study the $Q$ rather than the $\varphi$ fields since the former react to the analogue of a random potential. The formalism is quite similar and in particular the dispersion relations are unchanged. Particles are then localised at positions where the potential is small, i.e. where $l$, the separation, is small and so the probability that they are close to each other is greater than on a regular lattice. To verify that there is an effective attraction we consider the four-point function at small $\omega$. The connected four-point function in momentum space to leading order (for the Q's) is

$$
G_{4}\left(k_{1}, k_{2}, k_{3}, k_{4}, \omega\right)=\delta\left(\sum_{1}^{4} k_{l}\right) \frac{3 \omega^{2}}{\Pi_{1}^{4}\left(k_{i}^{2}+\omega\right)}
$$

indicating a positive $\left(\boldsymbol{Q}^{2}\right)^{2}$ term in the effective action with a coefficient of order $\omega^{2}$. Recall that in the usual case this term has a negative sign corresponding to repulsion among scalar particles. Equation (86) is nothing but a restatement that for small $\omega$

$$
\begin{aligned}
\left\langle\exp \left(-\frac{1}{2} \omega l \boldsymbol{Q}^{2}\right)\right\rangle & \sim \exp \left(-\frac{1}{2} \omega \boldsymbol{Q}^{2}\right)\left[1+\frac{1}{8}\left\langle z^{2}\right\rangle \omega^{2}\left(\boldsymbol{Q}^{2}\right)^{2}+\ldots\right] \\
& \sim \exp \left[-\frac{1}{2} \omega \boldsymbol{Q}^{2}+3 \omega^{2}\left\langle z^{2}\right\rangle\left(\boldsymbol{Q}^{2}\right)^{2} / 4 !+\ldots\right]
\end{aligned}
$$

and we should not be worried by the 'wrong' sign because of higher-order terms. 


\section{Acknowledgments}

It is pleasure to thank D Bessis for stimulating discussions on the subjects of $\S 6$.

Note added. After completing this work, we received the thesis of Dr Nieuwenhuizen (1983a). The results of $\$ \S 2-6$ appear in this work to a certain extent using a different notation. These results are partly published and partly to be published (Nieuwenhuizen $1980,1983 \mathrm{~b}, 1984$ ). He was able to give a more explicit solution to the differential recursion relation (48). We want to thank him for communicating his results and some references to us.

\section{References}

Alexander S, Bernasconi J, Schneider W R and Orbach R 1981 Rev. Mod. Phys. 53175

Casher A and Lebowitz J L 1971 J. Math. Phys. 121701

Christ N H, Friedberg R and Lee T D 1982a Nucl. Phys. B 20289

__ 1982b Nucl. Phys. B(FS)210 310, 337

Derrida B and Orbach R 1983 Phys. Rev. B 274694

Dyson F J 1953 Phys. Rev. 921331

Halperin B I 1965 Phys. Rev. A 139104

Itzykson C 1983 Fields on a random lattice in Proc. 1983 Cargése Summer School on Progress in Gauge Field Theory to be published

Lin T F 1970 J. Math. Phys. 111584

Nieuwenhuizen T M 1980 Physica 113A 173

— 1983a Thesis, Analytic Methods and Exact Solutions for One-dimensional Random systems, Rijksuniversiteit Utrecht

- 1983b Physica 120A 468

- 1984a J. Phys. A: Math. Gen. 171111

— 1984b Physica A submitted for publication

Stephen M J and Kariotis R 1982 Phys. Rev. B 262917

Thouless D J 1972 J. Phys. C: Solid State Phys. 577 